\title{
Prolonged balloon occlusion of the lower abdominal aorta during pelvic or sacral tumor resection
}

\author{
Yi Luo ${ }^{1}$, Mingyan Jiang ${ }^{2}$, Jianguo Fang ${ }^{1}$, Li Min ${ }^{1}$, Yong Zhou ${ }^{1}$, Fan Tang ${ }^{1}$, Minxun Lu ${ }^{1}$, Yitian Wang ${ }^{1}$, \\ Hong Duan ${ }^{1}$, Chongqi Tü ${ }^{1}$ \\ ${ }^{1}$ Department of Orthopedics, West China Hospital, Sichuan University, Chengdu, China; ${ }^{2}$ West China School of Stomatology, Sichuan University, \\ Chengdu, China \\ Contributions: (I) Conception and design: Y Luo, C Tu; (II) Administrative support: W Zhang, H Duan, C Tu; (III) Provision of study materials or \\ patients: Y Luo, M Jiang; (IV) Collection and assembly of data: M Jiang, M Lu, Y Wang; (V) Data analysis and interpretation: L Min, Y Zhou, F \\ Tang; (VI) Manuscript writing: All authors; (VII) Final approval of manuscript: All authors. \\ Correspondence to: Chongqi Tu. Department of Orthopedics, West China Hospital, Sichuan University, Chengdu 610041, China. \\ Email: tuchongqi@163.com.
}

Background: To explore the efficacy of lower abdominal aorta balloon occlusion technology in pelvis and sacral tumor surgery and to assess the safety of prolonged balloon occlusion.

Methods: From January 2008 to January 2017, 81 patients were diagnosed with sacrum or pelvic tumor and underwent surgery in our institution. Balloon catheters were placed through the femoral artery to occlude the abdominal aorta of the pelvic tumor and sacrum region undergoing tumor resection. These patients were divided into two group based on single balloon blocking time. Group A had a balloon blocking time of 60 minutes or less, and group B had a balloon occlusion time greater than 60 minutes. The patients in the two groups were compared with regards to operation time, intraoperative blood loss, blood transfusion volume, average length of hospital stay, and postoperative complications.

Results: No balloon displacement or leakage of the abdominal aorta occurred during the operations. The difference in operation time between the two groups was statistically significant $(\mathrm{P} \leq 0.05)$, and the differences in intraoperative blood loss, blood transfusion volume, and average hospital stay between groups A and B were not significant $(\mathrm{P}>0.05)$. The incidence of postoperative complications was $12 \%$ in group A, and $22.6 \%$ in group $\mathrm{B}$, with no statistically significant differences $(\mathrm{P}>0.05)$.

Conclusions: Prolonged balloon occlusion was safe and effective in the surgical treatment of complicated pelvic and sacral tumors. It did not increase the incidence of postoperative complications such as distal limb paralysis, arterial thrombosis, or ischemic necrosis.

Keywords: Sacral tumor; pelvic tumor; balloon occlusion; abdominal aorta; complications; safety

Submitted Dec 11, 2020. Accepted for publication Feb 08, 2021.

doi: $10.21037 /$ atm-21-138

View this article at: http://dx.doi.org/10.21037/atm-21-138

\section{Introduction}

Pelvic and sacral tumors contain large soft tissue masses that can invade the peripheral nerves, blood vessels, pelvic organs, and other important structures. Surgical resection is delicate with high risks and significant blood loss, which can be more than $10,000 \mathrm{~mL}(1,2)$. Intraoperative massive hemorrhage often leads to a lack of clarity in the surgical field, which affects the protection of important structures in the surgical area and the detection of tumor boundaries, prolongs the duration of the operation time, and increases the incidence of perioperative complications. Low abdominal aortic balloon occlusion has been widely used in the surgical resection of pelvic and sacral tumors (3-5). Current guidelines suggest that the occlusion time must be strictly controlled to within 60 minutes, otherwise complications of distal limb ischemic necrosis, 
limb numbness, and pain can easily occur $(6,7)$. However, 60 minutes is often insufficient to completely remove the tumor and intermittent opening of the balloon for 10 to 15 minutes increases the risk of intraoperative bleeding and tumor cell proliferation. From January 2008 to January 2017, 81 patients who met the research criteria in our hospital underwent abdominal aortic balloon occlusion technology to control intraoperative bleeding. The occlusion time was extended to varying degrees, with a high tumor resection rate and satisfactory efficacy. We present the following article in accordance with the STROBE reporting checklist (available at http://dx.doi.org/10.21037/ atm-21-138).

\section{Methods}

\section{Research patients}

Patients who were diagnosed with pelvic or sacral tumors in the Department of Orthopedics in the West China Hospital of Sichuan University from January 2008 to January 2017 were included in this retrospective study. Patients who underwent intraoperative abdominal aortic balloon occlusion with precise postoperative pathological diagnosis and complete case records were included. The cohort was divided into two groups based on the duration of the intraoperative balloon occlusion. Group A had a single balloon occlusion time of 60 minutes, and group B had a single balloon occlusion time greater than 60 minutes. Balloon occlusion time is recorded from the time of initial occlusion, which is defined as the completion of balloon filling to the first release of the intraoperative balloon. All procedures performed in this study involving human participants were in accordance with the Declaration of Helsinki (as revised in 2013). The study was approved by ethics board of West China hospital. Informed consent is waived due to the retrospective nature of this study.

\section{Balloon placement}

Preoperative vascular examination was performed to determine the renal artery opening position, and the balloon was placed according to conventional techniques following occlusion of the abdominal aorta. The operation commenced after the patient's central rate and blood pressure was stabilized. Systemic heparinization was performed with $0.5 \mathrm{mg} / \mathrm{kg}$ heparin, and $0.25 \mathrm{mg} / \mathrm{kg}$ heparin was added every 1 hour thereafter. In general, the first balloon occlusion would not be released until the tumor boundary had been treated and the tumor had been completely removed. After the ligation or suture of the bleeding site, the wound cavity was filled with common gauze or oil gauze, and then the balloon was released. The wound surface was treated again after 10-15 minutes. If the tumor was large and the surrounding anatomical boundaries were complex, the wound cavity would be filled with oil gauze or gauze during the 1-hour block, and then the balloon would be opened for 10 to 15 minutes before the second occlusion. Infusion speed was accelerated when the balloon was loosened to avoid a sudden drop in circulating blood volume as sudden unblocking of the abdominal aorta may result in a large amount of blood flowing into the lower body, which may result in transient blood pressure fluctuations. After the operation, the femoral artery incision was exposed, the balloon catheter was removed, and the incision was sutured after ensuring normal proximal blood ejection and distal blood return at the puncture point. If the puncture site was not bleeding well, the thrombus was removed by a Fogarty thrombus catheter at the distal and proximal ends of the artery. Intact blood supply of the lower limbs was confirmed prior to suture. Simultaneously, protamine and heparin were used, and the dorsal foot pulse of the catheter-placed side was closely monitored after the operation.

\section{Outcome indicators}

Outcome indicators were assessed, including first balloon occlusion time, surgery duration, intraoperative blood loss, blood transfusion volume, postoperative drainage volume, and total hospital stay (8-10). Postoperative complications including wound infection, joint dislocation, nerve dysfunction, iliofemoral arteriovenous thrombosis, rectal injury, and bladder injury were monitored. Surgical time refers to the time when the tumor site is cut open from the skin, and the tumor is completely removed, to the end of skin suture, excluding the time taken for balloon placement or removal.

\section{Statistic analysis}

Data were analyzed using the SPSS v17.0 software and expressed as mean \pm standard deviation $\left(\bar{x}_{ \pm s}\right)$. Analysis of variance (ANOVA) was used to compare groups of measurement data, and the chi-square test was used for comparison between groups of counting data. Results were 
Table 1 A comparison of the basic characteristics of patients in Group A and B

\begin{tabular}{|c|c|c|c|c|}
\hline Characteristics & Group A (50 cases) & Group B (31 cases) & Statistical magnitude & $P$ values \\
\hline Male & 22 & 17 & & \\
\hline Female & 28 & 14 & & \\
\hline Age & $42.32 \pm 14.42$ & $41.29 \pm 10.28$ & $\mathrm{t}=0.346$ & 0.730 \\
\hline Tumor location & & & $\chi^{2}=0.825$ & 0.364 \\
\hline Sacral tumor & 13 & 11 & & \\
\hline Pelvic tumor & 37 & 20 & & \\
\hline Tumor type & & & $\chi^{2}=0.535$ & 0.991 \\
\hline Chondrosarcoma & 9 & 4 & & \\
\hline Giant-cell tumor of bone & 8 & 6 & & \\
\hline Metastatic carcinoma & 7 & 5 & & \\
\hline Other & 2 & 1 & & \\
\hline
\end{tabular}

considered statistically significant when $\mathrm{P}<0.05$.

\section{Results}

\section{Basic patient characteristics}

A total of 95 patients were identified as potential candidates, 81 of whom met the inclusion criteria, including 50 cases in group A and 31 cases in group B. There were 39 males and 42 females in total, with an average age of 42.9 years. There were no significant differences $(\mathrm{P}<0.05)$ in the basic characteristics of the patients. The follow-up time ranged from 7 to 76 months post-surgery, with an average duration of 36.5 months. According to tumor types, there were 23 cases of chordoma, 16 cases of osteosarcoma, 13 cases of chondrosarcoma, 14 cases of giant-cell tumor of bone, 12 cases of metastatic carcinoma, and three cases of other tumor types. The basic characteristics of the patients in both groups were consistent, as shown in Table 1 .

\section{Surgical results}

No intra-or postoperative deaths were recorded among the 81 patients. In all cases, the balloon was well fixed during the operation without any arterial blood leakage. The mean operating time was 220.4 minutes, intraoperative blood loss was $1,259.9 \mathrm{~mL}$, blood transfusion was $511.1 \mathrm{~mL}$, postoperative drainage volume was $257.8 \mathrm{~mL}$, and the mean hospital stay was 10.32 days. There were no significant differences in operating time, blood loss, blood transfusion, and postoperative drainage volume between the two groups $(\mathrm{P}>0.05)$. Six patients in group $\mathrm{A}$, and seven patients in group B presented with postoperative complications. However, the difference between the two groups was not significant $(\mathrm{P}>0.05$; Table 2).

\section{Discussion}

\section{The application of intra-aortic balloon occlusion in the abdominal aorta}

Extensive resection of invasive or malignant tumors of the pelvis and sacrum has been clinically identified. However, as the tumor is often located adjacent to large blood vessels and nerves, resection surgery is complicated by the complex anatomical structure surrounding the tumor, significant intraoperative blood loss, and prolonged resection time (11). Therefore, successful control of intraoperative hemorrhage facilitates the efficacy of the surgery and minimizes risk to patients with pelvic and sacral tumors. Intraoperative low- 
Table 2 A comparison of the surgical results between group A and group B

\begin{tabular}{|c|c|c|c|c|}
\hline Outcome indicators & Group $A(N=50)$ & Group B (N=31) & $\mathrm{t} / \chi^{2}$ value & $P$ value \\
\hline Operation duration (min) & $214.40 \pm 52.32$ & $230.16 \pm 45.69$ & -1.382 & 0.171 \\
\hline Intraoperative blood loss (mL) & $1,205.20 \pm 352.36$ & $1,348.23 \pm 382.42$ & -1.719 & 0.090 \\
\hline Blood transfusion volume $(\mathrm{mL})$ & $464.00 \pm 388.49$ & $587.10 \pm 342.29$ & -1.449 & 0.151 \\
\hline Average hospital stay (d) & $10.06 \pm 1.58$ & $10.74 \pm 1.77$ & -1.801 & 0.076 \\
\hline Postoperative complications & & & $\chi^{2}=1.465$ & 0.690 \\
\hline Dysneuria & 2 & 2 & & \\
\hline Thrombosis of the iliac (femoral) artery & 0 & 1 & & \\
\hline Wound infection & 3 & 2 & & \\
\hline Other & 1 & 2 & & \\
\hline
\end{tabular}

pressure anesthesia control or ligation of one or both internal iliac arteries before tumor resection to reduce intraoperative bleeding is now rarely used in clinical practice. Although preoperative interventional embolization of tumor blood vessels can significantly reduce intraoperative bleeding, this often requires two surgeries as embolization of multiple blood supply arteries is necessary to achieve good results. This is both time-consuming and expensive, and therefore, has limited clinical application $(12,13)$.

Balloon catheters were initially used to dilate narrow digestive or arterial vessels or assist in the surgical management of abdominal aortic aneurysms. Later, it was gradually introduced into orthopedics to reduce intraoperative bleeding by blocking the flow of blood. While balloon catheters have been widely used for the surgical resection of pelvic and sacral tumors, it is now increasingly used in many types of pelvic surgery with a high risk of bleeding, such as placental previa cesarean section and pelvic trauma $(4,11,14,15)$. This method is easy to operate, significantly reduces intraoperative bleeding, and has no effect on blood supply to the heart, lung, brain, and other important organs and venous reflux. In this study, the vital signs of all the patients, including intraoperative central rate, blood pressure, and oxygen saturation, remained stable. The blood loss was $165-5,430 \mathrm{~mL}$, with an average of $1,096.67 \pm 796.68 \mathrm{~mL}$. This amount of surgical blood loss was significantly reduced compared to previous studies $(2,4,16)$.

\section{Research progress of balloon occlusion time}

Previous studies have generally considered that single intraaortic balloon occlusions should be limited to 60 minutes, and discontinuous balloon release is required to restore blood flow for 10 to 15 minutes when the operating time is prolonged. Otherwise, it may increase the risk of distal limb ischemic necrosis or even induce systemic multiorgan dysfunction $(5-7,17)$. Ullmark et al. (18) applied balloon occlusion technology to the lower extremity hip and knee joints, and the longest time of the single balloon occlusion was more than 3 hours. One patient suffered from cooling of the extremities and another patient suffered from necrotic ulceration of the toes caused by micro thrombus embolization of distal limb vessels. However, following the surgery, the blood supply to the lower extremities returned to normal in all patients. This may be related to the fact that the blockage of the plane was only in the common iliac artery or femoral artery, and the affected blood supply area did not involve important organs, and therefore part of the collateral circulation remained intact.

The tumors examined in this study were located in a problematic area, adjacent to the large pelvic vessels with a rich blood supply. It is difficult to control bleeding effectively with traditional electrocoagulation or sutures. 
The differences in the time and duration of surgery between the two groups were mainly due to varying surgical difficulties, including the boundary of the tumor, adhesion to important surrounding structures, and the nature of the tumor. The difference in operation time between the two groups was statistically significant, and the differences in intraoperative blood loss, blood transfusion volume, and average hospital stay between groups A and B were not significant. The duration of the occlusion depends on complete removal of the tumor. However, if the duration of occlusion is considerably prolonged, the balloon should be opened intermittently. In our opinion, any increased risk of complications caused by the proper extension of balloon occlusion time may be far less than the harm likely to be caused by extensive intraoperative bleeding (19-21).

\section{Matters requiring attention during balloon placement}

To prevent the risk of postoperative complications following implantation of the balloon, routine examinations should be performed preoperatively to fully appreciate the situation of the femoral artery, external iliac artery, and abdominal aorta. In particular, the elderly, or patients with hyperlipidemia and diabetes, should be thoroughly evaluated to exclude vascular lesions and atherosclerotic plaques to avoid any vascular injury or even vascular rupture and bleeding after the placement of the balloon catheter $(19,22)$. At present, there have been no reported cases of vascular wall damage caused by balloon compression, and this may be related to the fact that the balloon catheter was designed to expand the stenosis artery, and therefore, the pressure is likely to be relatively uniform.

Balloon catheters must be placed further away from the renal artery to prevent acute ischemic renal failure by blocking the supply of renal arterial blood $(16,23)$. The level of balloon filling also affects the blocking effect. Theoretically, if the filling is excessive, the arterial wall may be damaged, and balloon rupture may occur. In this study cohort, the balloon placement process was uneventful, and the dose of balloon contrast agent used ranged from $7-10 \mathrm{~mL}$ (5). The dose was determined by the presence of a dorsal foot artery pulse after occlusion, blood flow signal as observed by ultrasound, and balloon catheter displacement or balloon rupture during the operation.

In this study, a total of 4 patients developed femoral artery thrombosis, which was manifested as a weak dorsal foot pulse when the balloon catheter was removed. Intraoperative B-scan ultrasound indicated that the blood flow signal of the distal femoral artery on the side of the catheter was weakened, indicating partial thrombosis within the femoral artery. After conventional thrombosis, the thrombus was primarily located at the distal end of the catheter. The blood vessel was then recanalized, and a strong dorsal foot artery pulse could be detected. There were no cases of distal limb ischemic necrosis caused by low arterial blood flow after performing postoperative vasodilatation and blood circulation therapy. In general, although an appropriate extension of balloon occlusion time may lead to iliofemoral artery thrombosis, after removal of the thrombus, the risk of ischemic necrosis in the limb was not increased, and the risk of significant ischemia-induced bladder, rectum, and other injuries was also not increased. However, extension of balloon occlusion time can fully expose the tumor surgical site, ensure the complete resection of the tumor, and reduce the risk of postoperative recurrence caused by tumor cell contamination resulting from the blood-oozing wound. Therefore, the benefits of extended balloon occlusion time outweigh the disadvantages. This study suggests that under close monitoring, the time of balloon occlusion can be customized, thereby maximizing the use of this technology to aid clinical practice and benefit patients.

This report demonstrated that the selective use of intra-aortic balloon occlusion in the surgical treatment of pelvic and sacral tumors can significantly reduce bleeding, minimize the need for blood transfusions, and decrease medical costs. Prolonged balloon occlusion to control bleeding allowed for improved exposure of the surgical field, ensured the safety of the operation, and did not increase the incidence of postoperative complications such as distal limb paralysis or ischemic necrosis.

\section{Acknowledgments}

Funding: This work was supported by the Support Program for Science and Technology of Sichuan Province [2017SZ0106].

\section{Footnote}

Reporting Checklist: The authors have completed the STROBE reporting checklist. Available at http://dx.doi. org/10.21037/atm-21-138

Data Sharing Statement: Available at http://dx.doi. org/10.21037/atm-21-138 


\section{Page 6 of 7}

Conflicts of Interest: All authors have completed the ICMJE uniform disclosure form (available at http://dx.doi. org/10.21037/atm-21-138). The authors have no conflicts of interest to declare.

Ethical Statement: The authors are accountable for all aspects of the work in ensuring that questions related to the accuracy or integrity of any part of the work are appropriately investigated and resolved. All procedures performed in this study involving human participants were in accordance with the Declaration of Helsinki (as revised in 2013). The study was approved by ethics board of West China hospital. Informed consent is waived due to the retrospective nature of this study.

Open Access Statement: This is an Open Access article distributed in accordance with the Creative Commons Attribution-NonCommercial-NoDerivs 4.0 International License (CC BY-NC-ND 4.0), which permits the noncommercial replication and distribution of the article with the strict proviso that no changes or edits are made and the original work is properly cited (including links to both the formal publication through the relevant DOI and the license). See: https://creativecommons.org/licenses/by-nc-nd/4.0/.

\section{References}

1. Simpson AH, Porter A, Davis A, et al. Cephalad sacral resection with a combined extended ilioinguinal and posterior approach. J Bone Joint Surg Am 1995;77:405-11.

2. Zhang ZY, Fu CF, Yang YX, et al. Long-term outcomes following en bloc resection for sacral tumor: A retrospective analysis of 93 cases. Orthopedics 2011;34:e403-7.

3. Ratto N, Boffano M, Pellegrino P, et al. The intraoperative use of aortic balloon occlusion technique for sacral and pelvic tumor resections : A case-control study. Surg Oncol 2020;32:69-74.

4. Li D, Guo W, Tang X, et al. Preservation of the contralateral sacral nerves during hemisacrectomy for sacral malignancies. Eur Spine J 2014;23:1933-9.

5. Yang L, Tu CQ, Song HB, et al. Appling the abdominal aortic-balloon occluding combine with blood pressure sensor of dorsal artery of foot to control bleeding during the pelvic and sacrum tumors surgery. J Surg Oncol 2008;97:626-8.

6. Zhang L, Gong Q, Xiao H, et al. Control of blood loss during sacral surgery by aortic balloon occlusion. Anesth
Luo et al. Use of aortic balloon occlusion to decrease blood loss

Analg 2007;105:700-3.

7. Borger van der Burg BLS, van Dongen TTCF, Morrison $\mathrm{JJ}$, et al. A systematic review and meta-analysis of the use of resuscitative endovascular balloon occlusion of the aorta in the management of major exsanguination. Eur J Trauma Emerg Surg 2018;44:535-50.

8. Ji T, Guo W, Tang XD, et al. Reconstruction of type II+III pelvic resection with a modular hemipelvic endoprosthesis: a finite element analysis study. Orthop Surg 2010;2:272-7.

9. Tang X, Guo W, Ji T. Reconstruction with modular hemipelvic prosthesis for the resection of solitary periacetabular metastasis. Arch Orthop Trauma Surg 2011;131:1609-15.

10. Zhou Y, Duan H, Liu Y, et al. Outcome after pelvic sarcoma resection and reconstruction with a modular hemipelvic prostheses. Int Orthop 2011;35:1839-46.

11. Luo Y, Duan H, Liu W, et al. Clinical evaluation for lower abdominal aorta balloon occluding in the pelvic and sacral tumor resection. J Surg Oncol 2013;108:148-51.

12. Wirbel RJ, Roth R, Schulte M, et al. Preoperative embolization in spinal and pelvic metastases. J Orthop Sci 2005;10:253-7.

13. Domovitov SV, Chandhanayingyong C, Boland PJ, et al. Conservative surgery in the treatment of giant cell tumor of the sacrum: 35 years' experience. J Neurosurg Spine 2016;24:228-40.

14. Uchino H, Tamura N, Echigoya R, et al. REBOA - Is it really safe? A case with massive intracranial hemorrhage possibly due to endovascular balloon occlusion of the aorta (REBOA). Am J Case Rep 2016;17:810-3.

15. Bracale UM, Corte G, del Guercio L, et al. Endovascular treatment of abdominal aortic anastomotic pseudoaneurysm: The experience of two centers. Ann Ital Chir 2012;83:509-13.

16. Zhang Y, Guo W, Tang X, et al. Can aortic balloon occlusion reduce blood loss during resection of sacral tumors that extend into the lower lumber spine? Clin Orthop Relat Res 2018;476:490-8.

17. Mi C, Lu H, Liu H. Surgical excision of sacral tumors assisted by occluding the abdominal aorta with a balloon dilation catheter: a report of 3 cases. Spine 2005;30:E614-6.

18. Ullmark G, Hovelius L, Strindberg L, et al. Reduced bleeding through temporary balloon occlusion in hip and knee revision surgery. Acta Orthop Scand 2000;71:51-4.

19. Tang X, Guo W, Yang R, et al. Use of aortic balloon occlusion to decrease. J Bone Joint Surg Am 2010;92:1747-53. 
20. Akoz T, Yildirim S, Akan M, et al. Can indications for lower limb replantation and revascularization be expanded with simultaneous free-flap transfer for limb salvage? J. Reconstr. Microsurg 2004;20:621-9.

21. Hierner R, Betz AM, Comtet JJ, et al. Decision making and results in subtotal and total lower leg amputations: Reconstruction versus amputation. Microsurgery 1995;16:830-9.

22. Atar E, Bachar GN, Bartal G, et al. Use of peripheral cutting balloon in the management of resistant benign

Cite this article as: Luo Y, Jiang M, Fang J, Min L, Zhou Y, Tang F, Lu M, Wang Y, Duan H, Tu C. Prolonged balloon occlusion of the lower abdominal aorta during pelvic or sacral tumor resection. Ann Transl Med 2021;9(5):416. doi: 10.21037/ atm-21-138 ureteral and biliary strictures. J Vasc Interv Radiol 2005;16:241-5.

23. Forte DM, Do WS, Weiss JB, et al. Validation of a novel partial resuscitative endovascular balloon occlusion of the aorta device in a swine hemorrhagic shock model: Fine tuning flow to optimize bleeding control and reperfusion injury. J Trauma Acute Care Surg 2020;89:58-67.

(English Language Editor: J. Teoh) 